\title{
Hibridización de silúridos: una alternativa para la piscicultura con alto riesgo para la conservación de las especies nativas
}

\author{
Hybridization Silurids: An alternative for fish farming at high risk \\ for the conservation of native species.
}

\section{Hibridação Silurids: Uma alternativa para a criação de peixes em alto risco para a conservação de espécies nativas}

José A. Rodríguez-Pulido ${ }^{1}$, Pablo E. Cruz-Casallas ${ }^{2}$, Agustín Góngora-Orjuela $^{3}$

1 Biólogo, MSc, cPhD. Grupo de Investigación sobre Reproducción y Toxicología de Organismos Acuáticos (GRITOX), Instituto de Acuicultura, Grupo de Investigación en Reproducción y Genética Animal (GIRGA), Escuela de Ciencias Animales, Universidad de los Llanos, Villavicencio, Colombia.

2 MVZ, MSc, PhD. Grupo de investigación sobre Reproducción y Toxicología de Organismos Acuáticos (GRITOX), Instituto de Acuicultura, Universidad de los Llanos, Villavicencio, Colombia.

3 MV, MSc, PhD. Grupo de investigación en Reproducción y Genética Animal (GIRGA), Escuela de Ciencias Animales, Universidad de los Llanos, Villavicencio, Colombia.

Email: jarodriguez@unillanos.edu.co

Recibido: agosto 5 de $2015 \quad$ Aceptado: junio 2 de 2016

\section{Resumen}

El propósito de este artículo es analizar, desde una mirada crítica, el riesgo a que se ven expuestas ciertas especies de silúridos, sometidas a procesos de hibridación por razones estrictamente comerciales, incluyendo los efectos sobre el hábitat, la competencia y la erosión genética. Es fácil comprender que dichos procedimientos tendrán en el futuro serios efectos sobre los recursos hidrobiológicos, los servicios ecosistémicos y la seguridad alimentaria, los cuales sólo pueden ser medidos a través del tiempo y no de forma inmediata.

Palabras clave: Acuicultura, fitness, erosión genética, peces nativos (recursos acuáticos).

\begin{abstract}
The purpose of this article was to analyze, from a critical perspective, the risk that would be exposed some silurid species in captivity, subjected to hybridization processes for strictly commercial reasons, including habitat effects, competition and genetic erosion. It is easy to understand that these procedures will be in the future effects on aquatic resources, ecosystem services and food security, which can only be measured over time and not immediately.
\end{abstract}

Keywords: Aquaculture, fitness, genetic erosion, native fish (aquatic resources).

\section{Resumo}

O objetivo deste artigo foi analisar, a partir de uma perspectiva crítica, o risco ao qual seriam expostas algumas espécies de silúridos em cativeiro, sujeitos a processos de hibridação por razões exclusivamente comerciais, incluindo os efeitos sobre 
o habitat, a competição e a erosão genética. É fácil compreender que esses procedimentos terão, no futuro, efeitos sobre os recursos hidrobiológicos, os serviços ecossistêmicos e a segurança alimentar, os quais somente podem ser medidos ao longo do tempo e não de maneira imediata.

Palavras-chave: Aquicultura, aptidão, erosão genética, peixes nativos (recursos aquáticos).

\section{Introducción}

El avance de la acuicultura en Colombia y en el mundo, representa una nueva alternativa para superar la deficiente oferta de proteína de origen animal, contribuyendo a la solución de los graves problemas sociales, tales como la falta de alimento para consumo humano, el desempleo y los bajos ingresos per cápita de las comunidades rurales.

En Colombia el consumo de peces provenientes de cultivo logró un incremento per cápita de aproximadamente $7 \mathrm{Kg}$ en los últimos años, variando de 0.7 kg en 1970 a 7.8 kg/año el 2008, llegando a igualar las estadísticas de la pesca de captura como fuente de pescado comestible. En el año 2015, la acuicultura continental tuvo una producción de 35 millones de toneladas, con un aumento de 0.4 millones en relación al año 2008 (Agricultura y Acuicultura 2012). La razón de dicho incremento, entre otras causas, se debe a la disminución de los recursos pesqueros, tanto marinos como continentales, no sólo por efecto de la sobre pesca, la pesca ilícita con mallas y dinamita, sino por la destrucción de sus hábitats como consecuencia de la deforestación de las cuencas, que ha aumentado la sedimentación de los ríos y desecación de humedales; así mismo, el uso excesivo de agroquímicos y pesticidas que terminan en los cuerpos de agua, amenazando los nichos de alimentación, reproducción y protección de muchas de las especies nativas y la alteración de ecosistemas por diversas obras hidráulicas (represas, diques, bocatomas, etc.) sin criterios ambientales.

La acuicultura continental de Colombia se reduce casi exclusivamente al cultivo de organismos foráneos como camarón blanco (Pennaeus vannamei), tilapia (Oreochromis sp.) y trucha arco iris (Oncorhynchus mykiss), siendo limitada la oferta de especies nativas como cachama (Piaractus brachypomus), yamú (Brycon amazonicus) y bocachico (Prochilodus mariae), productos que alcanzan mercados regionales, pero con un bajo nivel competitivo en la cadena piscícola y prácticamente sin alcanzar mercados internacionales.

Una alternativa, para diversificar la producción piscícola nacional es incorporar nuevas especies a los sistemas de cultivo, tal como se ha intentado ofreciendo alevinos de silúridos, para ampliar la oferta de especies para la producción piscícola. Actualmente muy pocas especies de bagres son cultivadas a escala comercial en Colombia, por lo que toda la carne de silúridos que se consume en el país proviene de la actividad pesquera.

Durante el desarrollo de la siluricultura se ha observado que algunas especies de silúridos, como por ejemplo el rayado (Pseudoplatystoma sp,) produce altos volúmenes de semen, mientras en otras especies no presentan esta misma característica; A pesar de esto algunos cruzamientos muestran mayor crecimiento que los parentales, debido posiblemente a la expresión del vigor hibrido.

En suramerica, Brasil ha sido uno de los países pioneros en el cruzamiento de especies de silúridos con fines comerciales, por ejemplo, en Mato Grosso, el cultivo de "Pintado Amazónico", hibrido de Pseudoplatystoma corruscans con Leiarius marmoratus, constituye el principal producto de la pisciultura de este estado, alcanzando una producción estimada de $16.144 \mathrm{~kg} / \mathrm{ha}$ y conversiones por debajo de 2, con rendimientos financieros del 33\% (comunicación. personal. Dr. Darci Carlos Fornari).

La pesca comercial en la cuenca binacional del Orinoco y particularmente en el Meta, está concentrada especialmente en grandes pimelónidos y carácidos, de los cuales Pseudoplatystoma faciatum (orinocoense), Pseudoplatystoma tigrinum, Colossoma macropomum y Piaractus brachypomus, son las especies que más se capturan, debido a la gran demanda que tiene su carne, lo cual determina precios altos en los mercados (Ajiaco-Martínez 2002).

Frente a la disminución de los stocks pesqueros, la inhabilidad para administrar los recursos acuáticos y el estado precario de la normatividad se hace necesario además de proteger los recursos, poder dar respuesta a las crecientes demandas por proteína de origen animal. Congruente con ello se deben instaurar políticas coherentes con las necesidades de las comunidades, de pesca artesanal y de la protección del recurso.

Una de las mayores limitaciones de los recursos pesqueros en la cuenca del Orinoco colombiano, es la poca disponibilidad de información sobre las especies cultivadas y con potencial para cultivo, lo cual impide 
tomar las mejores acciones para su manejo adecuado y efectivo aprovechamiento (Ramirez-Gil.H. 2010).

La introducción responsable de nuevas especies ícticas, con grandes potencialidades de cultivo y mercado, implica el conocimiento de su biología básica, adaptación al cautiverio, evaluación de su desempeño comercial en cultivo y aceptación por los consumidores (Rodríguez-Pulido 2011). La introducción de especies es considerada la segunda causa de pérdida de biodiversidad y se estima que la acuicultura es responsable del $40 \%$ de las introducciones, incluyendo organismos vivos modificados, e impactos ecológicos adversos (Gutiérrez-Bonilla et al., 2012). Debido principalmente a que se hace muy poco para evitar los escapes de ejemplares del cultivo a los ambientes naturales.

La Universidad de los Llanos ha ejecutado varios trabajos con bagre rayado (Pseudoplatystoma sp) y yaque (Leiarius marmoratus), tendientes a desarrollar un paquete tecnológico que permita introducir estas especies puras a los sistemas piscícolas; sin embargo, su marcada estacionalidad reproductiva y principalmente los hábitos alimenticios carnívoros, han dificultado su adaptación al consumo de concentrados comerciales, siendo mejor el desempeño productivo de yaque, aunque de esta especie aún no ha sido posible la producción masiva de alevinos (Cruz et al., 2010). En todo caso, aún falta generar paquetes tecnológicos que permitan optimizar la producción a escala comercial, tanto en sistemas de cultivo extensivos como intensivos, sin que afecten al ambiente, aplicando el principio precautelar (Hans 1985).

El vigor híbrido o ventaja del heterocigoto, puede optimizar la producción con mayores crecimientos, resistencia a enfermedades, por la combinación de virtudes de sus parentales, mediante la exogamia, sin embargo, de acuerdo con (Echelle et al., 2014) la mayoría de veces su ocurrencia, representa un riesgo para la conservación de las especies y generalmente requiere de estrategias en el manejo que eviten que los híbridos se puedan cruzar entre sí o con sus progenitores (empantanamiento genético) (Mallet 2005, Mallet 2007). Por ello, evaluar la maduración sexual y el potencial reproductivo de los híbridos se hace urgente, máxime que la experiencia brasilera hace muy atractivo su manejo y ante todo por la alta rentabilidad económica.

A las anteriores consideraciones es necesario sumar el riesgo a que quedan sometidas las comunidades que dependen económicamente de este recurso íctico. En el caso del río Meta, afluente del Orinoco, por tratarse de una cuenca binacional y como lo reportan Ajiaco-
Martínez (2002), más de 2400 personas ejercen la actividad pesquera, con un esfuerzo por unidad de pesca (UEP) que fluctuó entre los 5,7 y los $60 \mathrm{Kg} / \mathrm{UEP} /$ día, para los años 1995 a 2009. Los autores señalan que el $30 \%$ de este esfuerzo pesquero está representado por las capturas de bagre rayado (Pseudoplatystoma sp.). En otros países, el pescado constituye fuente primaria de proteína, vitaminas, minerales y ácidos grasos esenciales, aportando el $30 \%$ de la dieta de Asia, el $20 \%$ en África, $10 \%$ en América Latina y el Caribe, el $7 \%$ en América del Norte y el $10 \%$ de Europa. En ellos, tanto pescadores artesanales, pequeños campesinos como las comunidades indígenas, consumen parte del recuso y venden al exterior de sus áreas de pesca sus productos (Agricultura y Acuicultura 2012).

\section{Hibridación}

El término híbrido se define como el descendiente de individuos de constitución genética distinta. También se refiere comúnmente a hijos de padres de especies u otras categorías taxonómicas diferentes; pero en rigor, es el descendiente de padres de distinta constitución genética (genotipos), independientemente de su categoría taxonómica. Para establecer su ocurrencia es necesario evidenciar cuándo realmente hay dos especies que se cruzan para originar una población intermedia (Echelle et al., 2014, Stone 2000). Seehausen (2004), mostró el papel de la hibridación en la evolución adaptativa y la especiación, como determinante de la formación de especies, por ello es crucial determinar cuando el agente causal de dicha especiación es natural y cuando es responsabilidad del hombre (Allendorf et al 2001).

\section{Hibridación natural}

Mallet (2005) demostró el papel de la hibridación en la formación de nuevas especies, difiriendo de los primeros evolucionistas, quienes la veían como una amenaza al aislamiento y especiación, ya que como zoólogos consideraban necesario el aislamiento del flujo génico para poder mantener las especies (Dobzhansky et al., 1968, Lenormand 2002, Mayr 1989).

La hibridación intraespecífica es ampliamente aceptada por botánicos, a pesar que el flujo de genes puede reducir la habilidad para que la población se adapte a las condiciones locales. Mallet (2005) Estableció que entre un 6 y $10 \%$ de hibridación ocurre en poblaciones animales y es de gran peso en el fenómeno de islas. Generalmente híbridos interespecíficos suelen darse en especies que divergen alopátricamente y por algún evento se encuentran luego en simpatría. 


\section{Hibridación antropogénica}

Las tasas de hibridación antropogénica han aumentado drásticamente, no sólo de manera intencionada, sino además por las presiones a las que se someten las especies naturales, tales como la introducción de otros ejemplares, fragmentación y modificación de sus hábitats.

Si bien la hibridación es una herramienta de la especiación, saber cuándo ésta se da en condiciones naturales y cuando es influenciada por la actividad humana es cada vez más difícil por la permanente introducción de especies, las fuertes alteraciones ecosistémicas y la manipulación de especies.

\section{Híbridos como herramienta de mejoramiento genético}

La hibridación puede contribuir a la disminución y eventual extinción de especies de dos formas generales:

- A causa de los híbridos estériles o parcialmente estériles. Resultados de hibridación en la pérdida de la capacidad de reproducción y pueden reducir la tasa de crecimiento de la población por debajo del necesario para el reemplazo (empantanamiento demográfico).

- En el caso de híbridos fértiles, genéticamente distintas poblaciones se pueden perder a través de la mezcla genética.

Aunque no se presente introgresión genética, los híbridos disminuyen la capacidad reproductiva de la población, como se ha visto con la introducción a Europa de machos de visón americano o en el caso de la trucha toro en el parque nacional de Oregon, (Solberg et al., 2014). En ambos casos, como en el caso de la mula, los productos del hibridizaje son estériles pero disminuyen la posibilidad de que las poblaciones naturales continúen con tasas de reproducción altas, pues ya sea por abortos o por no poder cruzarse con su especie.

En muchos casos la hibridación además conlleva a introgresión génica y se producen enjambres de híbridos que desplazan las poblaciones naturales (Šimková et al., 2015), como el caso de las abejas africanizadas, o en el caso de truchas y bagres donde se originó un desplazamiento espacial a las poblaciones nativas,(Prado et al., 2012) y pueden ser más exitosos los híbridos en los ambientes intervenidos, desplazando a los grupos parentales.

\section{Hibridación en piscicultura}

Se estima entre un 6 y un $10 \%$ los cruces inter-específicos en vertebrados (Mallet 2005, Prado et al., 2012), en ambientes pantanoso (Seehausen 2004), se reportan como eventos comunes entre el 4 y $5 \%$, y que además como fuente de variabilidad genética y base evolutiva de las especies (Alves AL. 2014). En acuicultura el uso de híbridos en los cultivos comerciales es mayor, buscando en el hibrido mejor desempeñó y resistencia a bajas temperaturas, que en las especies parentales, más de 8.000 toneladas en híbridos de cachama (Mylossoma, Colossoma y Piaractus) (Hashimoto et al., 2010); 1,1 billones de Kg de tilapias cuyo $30 \%$ de híbridos buscan poblaciones infértiles o de solo machos, como el cruce entre Oreochromis niloticus X O. aureus. En Tailandia se producen 136 millones de kg de bagres híbridos de Clarias gariepinus $X$ C. macrocephalus cuyos ejemplares además de tener mejores crecimiento resultaron resistentes a enfermedades (Hashimoto et al., 2010), mejor calidad de carne (Alves AL. 2014), mayores rendimientos económicos (de Verdal et al., 2014, Dunham et al., 2014). En Acuariofilia el uso de cruces y producción de híbridos es más común, buscando variaciones en forma y color. Recientemente se encontró que los híbridos Pseudoplatystoma carruscans con Leiarius marmoratus aceptan los alimentos balanceados comerciales y presentan menor canibalismo.

En el caso de los peces, los híbridos producidos en criadero son un problema importante de conservación para muchas especies. Por ejemplo, hasta dos millones de salmón del Atlántico se estiman que escapan de las granjas de salmón cada año. Todos los híbridos (f1, f2 y retrocruces) de animales de la granja con peces silvestres muestran reducción de la supervivencia en comparación al salmón salvaje (de Eyto et al., 2015, Jackson et al., 2015). El hecho de tener una supervivencia global baja significa que esto se traduciría en el reclutamiento reducido en la población, en su adaptabilidad acumulativa y con ello llevando al vórtice de extinción a las poblaciones salvajes (Senanan et al., 2004)

\section{Eficacia biológica por hibridación y sus consecuencias en conservación}

Si bien la hibridación nos promete mayores ganancias económicas por las ventajas del vigor hibrido (heterosis), no se muestra como dichas diferencias en el número de cromosomas o de genes puede reducir drásticamente el fitness de las poblaciones naturales, llevar a los híbridos a no poder constituir parejas (Burton et al., 2007) o a pérdidas de genes por introgresión (Roca et al., 2004). Además pone en dificultades a conservacionistas a la hora de decidir cuales ejemplares son dignos de conservar y se coloca en riesgo no solo 
a las poblaciones naturales sino a todos aquellos que dependen de servicios ecosistémicos de la población natural (Masoudi et al., 2016), para nuestro caso las comunidades de pescadores e indígenas serán los primeros en verse afectados.

\section{Mecanismos de identificación de híbridos}

Datos morfológicos de estructuras como los otolitos permiten comúnmente caracterizar especies (Masoudi et al., 2016). Sin embargo, existen dificultades en la identificación temprana de peces (alevinos) y más aún cuando se trata de híbridos, por cuanto difícilmente los análisis multivariados son suficientes para la identificación de estos animales. Por ello, se hace necesario aplicar técnicas que permitan tanto la identificación temprana como la identificación correcta de híbridos, como son las técnicas moleculares. Entre las alternativas podemos citar el análisis de cariotipos, la citometria de DNA (Xiao et al 2014)o el uso de técnicas moleculares rápidas y certeras, como los RFLPs (polimorfismos de longitud de fragmentos de restricción) o SNPs (polimorfismos de un solo nucleótido), el uso de micro- satélites, códigos de barra genéticos (DNA barcode), PCR en tiempo real y/o, buscar genes candidatos responsables de determinados fenotipos (pirosecunciación) (Hashimoto et al., 2010, Hasselman et al., 2014, Ribas et al., 2016).

\section{Normatividad en Colombia que regula la conservación de Especies Hidrobiológicas}

Los recursos naturales hacen parte del patrimonio público, es obligación del Estado y de las personas proteger las riquezas culturales y naturales de la Nación (Constitución PT. Colombia arte. VIII), asimismo el "Estado planificará el manejo y aprovechamiento de los recursos naturales, para garantizar su desarrollo sostenible, su conservación, restauración o sustitución" (Constitución PT. Colombia arte. LXXX). En razón a estos postulados constitucionales se genera un desarrollo normativo para la protección y conservación de la fauna y los recursos biológicos de las aguas como un recurso natural renovable, en tanto se han determinado los factores que deterioran el ambiente, entre ellos la extinción o disminución cuantitativa o cualitativa de especies animales o vegetales o de recursos genéticos, así desde los mismos Código Nacional de Recursos Naturales Renovables y de Protección al Medio Ambiente, se determinó la necesidad de requerir autorización especial a la importación, producción, venta o expendio de hibridos o nuevas especies logradas mediante el uso de recursos genéticos (Decreto 2811, 1974) disposición que ha sido parcialmente desarrollada.
Mediante Decreto 1681 de 1978 se reglamenta la parte X del libro II del Decreto - Ley 2811 de 1974, que trata de los recursos hidrobiológicos, y parcialmente la Ley 23 de 1973, para lo cual se determina que el cultivo de peces es una acción relacionada con la pesca, que debe ser entendida como aquella actividad que tiene por objeto la producción de organismos hidrobiológicos, término simple que se ha quedado corto para definir las complejidades de la producción hidrobiológica de estas especies. En la actualidad se ha generado una discusión entre principios económicos y ambientales que también se han traslado a los recursos hidrobiológicos, particularmente a los peces, pues la necesidad de generar mayor proteína, con la producción de especies a un menor tiempo y costo, ha generado que se investigue genética y biológicamente la aceleración de las fases de producción, pero surgen cuestionamientos y múltiples preocupaciones respecto a los riesgos que implica la modificación genética o el cruce biológico de especies en el contexto ambiental, pues ello representaría un riesgo para otros individuos y la sostenibilidad ambiental de los ecosistemas.

Respecto a los compromisos internacionales, el convenio de diversidad biológica aprobado mediante la Ley 165 de 1994, tiene como objetivo la conservación de la diversidad biológica, la utilización sostenible de sus componentes y el acceso adecuado a esos recursos y una transferencia apropiada de las tecnologías pertinentes, teniendo en cuenta todos los derechos sobre estos recursos y a esas tecnologías, a la luz de este convenio las especies domesticadas o cultivadas se entienden como especies en cuyo proceso de evolución han influido los seres humanos para satisfacer sus propias necesidades, sin dejar de lado su sostenibilidad y el equilibrio de los ecosistemas.

La complejidad de los organismos modificados o con variaciones por su cruce biológico, son múltiples y aún desconocidas en distintos aspectos, pues los avances científicos actuales no han permitido determinar con total certeza las consecuencias y las variaciones que se puedan generar en los ecosistemas y en las demás especies por la introducción de especies exóticas, para ello es fundamental la protección de la biodiversidad, por lo cual, los estados vinculados al convenio de diversidad biológica, deberán propender por la conservación in situ, evitando cualquier amenaza a las especies nativas, en tanto que deberán establecer o mantener medios para regular la utilización y liberación de organismos vivos modificados a los ecosistemas naturales, evitando así daños irreversibles a las especies nativas y a su hábitat. 
Teniendo claridad en que es una obligación común entre el estado y los particulares velar por la sostenibilidad de los recursos naturales renovables, se ha dispuesto que todo proyecto, obra o actividad, que genere afectación a los recursos naturales deberá contar con licencia ambiental, dependiendo de su grado de complejidad. El Decreto 2041 de 2014 determina expresamente las actividades que deberán contar con licencia ambiental y en la cual no prevé el desarrollo, cultivo o producción de peces híbridos por técnicas biológicas tradicionales, tan solo prevé la actividad de importar especies no nativas e híbridos, dejando un vacío normativo respecto al desarrollo de estas prácticas en el nivel nacional, donde eventualmente puede ser una actividad de riesgo para los recursos naturales y las demás especies dentro del equilibrio biológico del ecosistema. Esta falta de previsión normativa no es un permiso para el desarrollo de actividades de riesgo para el entorno, por lo cual el principio de precaución se constituye como una alternativa de protección frente a los recursos naturales que tengan probabilidad de sufrir un daño grave e irreversible.

El principio de precaución, es un instrumento estructural de protección ambiental y de manera particular de todos aquellos recursos naturales que se vean en riesgo ante actividades inciertas sobre los mismos, para ello la doctrina de manera general ha identificado unas condiciones mínimas, tales como la existencia de una amenaza o riesgo a la integridad ambiental, incertidumbre científica respecto al proyecto, obra o actividad y el deber de proteger el bien común mediante la evitación del daño (Cózar, 2005). En este mismo sentido hay quienes determinan dentro de los requisitos o condiciones de aplicación del principio precautorio, que no es suficiente la existencia de peligro de daño, sino que este debe ser grave e irreversible, pues es claro que toda actividad humana genera deterioro e impactos sobre los recursos naturales, de tal modo que no se integraría con los postulados del desarrollo sostenible.

La legislación ambiental colombiana adoptó el principio de precaución, el cual deberá ser aplicado tanto por las autoridades ambientales, como por los particulares cuando "exista peligro de daño grave e irreversible, la falta de certeza científica absoluta no deberá utilizarse como razón para postergar la adopción de medidas eficaces para impedir la degradación del medio ambiente" (Ley 99, 1993), la Corte Constitucional de Colombia se ha pronunciado frente a este principio en múltiples oportunidades indicando su importancia dentro del sistema normativo ambiental y ha determinado que su aplicación tampoco podrá ser deliberada, sino que deberá ser excepcional y motivada, cuando se cuente siquiera con el conocimiento de la existen- cia de un peligro de daño, que sea grave e irreversible, que exista principio de certeza científica, así no sea absoluta y que la decisión sea adoptada mediante acto administrativo debidamente motivado para impedir la degradación del medio ambiente (Corte Constitucional de Colombia, 2002). De este modo es claro que podrá ser aplicado este principio para el caso en estudio, sea por la decisión de los particulares de manera consciente o por la autoridad ambiental siempre que se cumpla con las condiciones antes referidas.

Por lo tanto al evidenciar un vacío normativo respecto al requerimiento de estudios y de licencia ambiental previo al desarrollo, producción y cultivo de especies hibridas de peces, se hace necesario motivar el desarrollo normativo al respecto, evidenciando un verdadero control y conocimiento de las autoridades públicas ambientales de los posibles impactos sobre los recursos naturales por el desarrollo de actividades de hibridación de especies icticas. Mientras ello sucede, el principio de precaución será un instrumento idóneo para defender la integridad ambiental y evitar así daños complejos a los recursos naturales que serán legadas a las futuras generaciones.

\section{Consideraciones finales}

Aplicando el principio precautelar se hace necesario verificar la conveniencia de hibridar las especies de silúridos y regular la producción de estas especies en cautiverio, así como restringir la ceba a ambientes controlados que eviten la fuga a los ambientes naturales, priorizando los intereses ambientales sobre los económicos para no tener que implementar medidas de remediación.

\section{Referencias}

Agricultura OdINUplAyl, Acuicultura ddPy. 2012. El estado mundial de la pesca y la acuicultura 2012. FAO

Ajiaco-Martínez R-GHyRE. 2002. La pesca en la Orinoquia Colombiana: Pasado, presente y futuro., ed. INdPyA INPA, pp. 23969. Ministerio de agricultura: Boletin cientifico

Alves AL. SVE, Vitti- Moro G, Ganeco-Kirschnik LN. 2014. Riscos genéticos da produção de híbridos de peixes nativos. Documentos:

Allendorf FW, Leary RF, Spruell P, Wenburg JK. The problems with hybrids: setting conservation guidelines. Trends Ecol Evol. 2001;16: 613-22

Burton RS, Byrne RJ, Rawson PD. Three divergent mitochondrial genomes from California populations of the copepod< i> Tigriopus californicus</i>. Gene. 2007;403:53-59

Cruz NE, Marciales LJ, Diaz Olarte JJ, Murillo Pacheco R, Medina Robles VM, Cruz Casallas PE. Desempeño productivo del yaque 
(Leiarius marmoratus Gill, 1870) bajo diferentes densidades de siembra en estanques en tierraa. Rev Colomb Cienc Pec. 2010;23:325-35

de Eyto E, White J, Boylan P, Clarke B, Cotter D, et al. The fecundity of wild Irish Atlantic salmon< $\mathrm{i}>$ Salmo salar $</ \mathrm{i}>\mathrm{L}$. and its application for stock assessment purposes. Fish Res. 2015;164:15969

de Verdal H, Rosario W, Vandeputte M, Muyalde N, Morissens P, et al. Response to selection for growth in an interspecific hybrid between Oreochromis mossambicus and O. niloticus in two distinct environments. Aquaculture. 2014;430: 159-65

Dobzhansky T, Ehrman L, Kastritsis P. Ethological isolation between sympatric and allopathic species of the $<\mathrm{i}>$ Obscura $</ \mathrm{i}>$ group of $<\mathrm{i}>$ Drosophila< $<\mathrm{i}>$. Anim Behav. 1968;16:79-87

Dunham RA, Ramboux ACR, Perera DA. 2014. Effect of strain on the growth, survival and sexual dimorphism of channel×blue catfish hybrids grown in earthen ponds. Aquaculture. 2014;420-421:S20-S24

Echelle AA, Schwemm MR, Lang NJ, Nagle BC, Simons AM, et al. Molecular systematics and historical biogeography of the $<\mathrm{i}>$ Nocomis biguttatus $</ i>$ species group (Teleostei: Cyprinidae): Nuclear and mitochondrial introgression and a cryptic Ozark species. Mol Phylogenet Evol. 2014;81:109-119

Gutiérrez-Bonilla FdP, Lasso-Alcalá C, Baptiste M, Sánchez-Duarte $\mathrm{P}$, Díaz A. 2012. VI Catálogo de la biodiversidad acuática exótica y trasplantada en Colombia: moluscos, crustáceos, peces, anfibios, reptiles y aves. Serie editorial Recursos Hidrobiológicos y Pesqueros Continentales de Colombia. Bogotá, DC: Instituto de investigación de los recursos biológicos Alexander von Humboldt $(\mathrm{IAvH})$

Hans J. 1985. Tecnica, Madicina y Etica (Sobre la practica del principio de responsabilidad). 206 pp.

Hashimoto DT, Mendonça FF, Senhorini JA, Bortolozzi J, de Oliveira C, et al. 2010. Identification of hybrids between Neotropical fish Leporinus macrocephalus and Leporinus elongatus by PCRRFLP and multiplex-PCR: Tools for genetic monitoring in aquaculture. Aquaculture 298: 346-49

Hasselman DJ, Argo EE, McBride MC, Bentzen P, Schultz TF, et al. Human disturbance causes the formation of a hybrid swarm between two naturally sympatric fish species. Mol Ecol. 2014;23:1137-1152

Jackson D, Drumm A, McEvoy S, Jensen $\varnothing$, Mendiola D, et al. A panEuropean valuation of the extent, causes and cost of escape events from sea cage fish farming. Aquaculture. 2015;436:21-26

Lenormand T. Gene flow and the limits to natural selection. Trends Ecol Evol. 2002;17:183-189

Mallet J. Hybridization as an invasion of the genome. Trends Ecol Evol. 2005;20:229-37

Mallet J. Hybrid speciation. Nature. 2007;446:279-283

Masoudi M, Esmaeili HR, Teimori A, Gholami Z, Gholamhosseini $A$, et al. Sympatry and possible hybridization among species of the killifish genus Aphanius Nardo, 1827 (Teleostei: Cyprinodontidae) in Southwestern Iran. Limnologica - Ecology and Management of Inland Waters. 2016;59:10-20

Mayr E. Speciational evolution or punctuated equilibria. J Soc Biol Struct. 1989;12:137-158

Prado FDd, Hashimoto DT, Senhorini JA, Foresti F, Porto-Foresti F. Detection of hybrids and genetic introgression in wild stocks of two catfish species (Siluriformes: Pimelodidae): The impact of hatcheries in Brazil. Fish Res. 2012;125:300-305

Ramírez-Gil H. REA-MyCB-R. 2010. Estado actual de la pesca del bocachico Prochilodus marie en la Orinoquia Colombiana. Informe final proyecto de investigacion IIOC_Unillanos., ed. Udl Llanos

Ribas L, Robledo D, Gómez-Tato A, Viñas A, Martínez P, Piferrer F. 2016. Comprehensive transcriptomic analysis of the process of gonadal sex differentiation in the turbot (Scophthalmus maximus). Mol Cell Endocrinol. 2016;422:132-49

Roca AL, Georgiadis N, O'Brien SJ. Cytonuclear genomic dissociation in African elephant species. Nat Genet. 2004;37:96-100

Rodríguez-Pulido JA. 2011. EL CULTIVO DE BAGRES PIMELODIDOS. Comunicaciones del III Coloquio de la Red de Investigación Sobre la Ictiofauna Amazónica-RIIA: 204

Seehausen O. 2004. Hybridization and adaptive radiation. Trends Ecol Evol. 2004;19:198-207

Senanan W, Kapuscinski AR, Na-Nakorn U, Miller LM. Genetic impacts of hybrid catfish farming $(<\mathrm{i}>$ Clarias macrocephalus $<$ / $\mathrm{i}>\mathrm{x}<\mathrm{i}>\mathrm{C}$. gariepinus $</ \mathrm{i}>$ ) on native catfish populations in central Thailand. Aquaculture. 2004;235:167-184

Šimková A, Vojtek L, Halačka K, Hyršl P, Vetešník L. The effect of hybridization on fish physiology, immunity and blood biochemistry: A case study in hybridizing Cyprinus carpio and Carassius gibelio (Cyprinidae). Aquaculture. 2015;435:381-389

Solberg MF, Zhang Z, Glover KA. 2014. Are farmed salmon more prone to risk than wild salmon? Susceptibility of juvenile farm, hybrid and wild Atlantic salmon $<\mathrm{i}>$ Salmo salar $</ \mathrm{i}>\mathrm{L}$. to an artificial predator. Applied Animal Behaviour Science

Stone G. Phylogeography, hybridization and speciation. Trends Ecol Evol. 2000;15:354-355

Xiao J, Kang X, Xie L, Qin Q, He Z, et al. The fertility of the hybrid lineage derived from female Megalobrama amblycephala x male Culter alburnus. Anim Reprod Sci. 2014;151:61-70

Ley 99. Diario oficial de la república de Colombia 4114, Bogotá, 22 de diciembre de 1993.

Corte Constitucional de Colombia. (23 de abril de 2002) Sentencia C-293 de 2002, expediente D-3748 [MP Alfredo Beltrán Sierra].

Decreto 2811 de 1974. Diario oficial de la república de Colombia 34243, Bogotá, 18 de diciembre de 1974.

Glosario de Términos Genéticos. National Human Genome Research Institute. http://www.genome.gov/sglossary.cfm. 